\title{
Myelolactoferrin Index (MLF) and Myelopoiesis During Bacterial Infections and Malignant Diseases*
}

\author{
R. Neth, J. Nowrath, M. Thomsen, C. Wiggers, M. Gabrecht, W. Rehpenning, K. Winkler, \\ U. Krause, H. Soltau, and H. Kraemer-Hansen
}

\begin{abstract}
The myelolactoferrin index (MLF) is significantly lowered in patients with bacterial infections and with malignant diseases. In both cases it can be assumed that the alteration of the granulopoietic storage pool is followed by a decrease in lactoferrin. Therefore, it seems advisable to study (a) to what extent the immunocytochemical quantitative evaluation of lactoferrin content of mature neutrophil granulocytes can be used to recognize any toxic damage caused to bone marrow cells by long-term therapies or environmental toxins and (b) whether this method is more sensitive than the conventional counting of granulocytes and thrombocytes.
\end{abstract}

\section{A. Introduction}

Myelopoiesis is regulated by cell-derived molecules that stimulate or inhibit proliferation and differentiation of granulocytemacrophage progenitor cells. The interactions of positive and negative feedback mechanisms regulate the production of granulocytes and macrophages and probably keep granulocytes and monocytes within the tightly controlled limits noted in vivo. Lactoferrin located in the secondary

* Universitätskliniken D-2000 Hamburg 20, Martinistrasse 52, FRG

Supported by the Deutsche Forschungsgemeinschaft and the Deutsche Krebshilfe granules of mature neutrophil granulocytes has been implicated as a negative feedback regulator of myelopoiesis [1]. Lactoferrin acts in vitro as an inhibitor of granulocyte and macrophage colony formation, and in vivo mouse granulopoiesis is decreased by lactoferrin [1]. However, there is no information available on a possible physiological role of lactoferrin on the regulation of myelopoiesis in humans. A correlation between the lactoferrin content of granulocytes and the bone marrow pool of these cells seems to indicate that lactoferrin may also function as a negative feedback regulator substance of myelopoiesis in the human. A reduction of the granulopoietic storage pool is known to occur during bacterial infections by way of increased cell turnover and during treatment of malignant diseases by way of drug-induced inhibition of myelopoiesis. We have, therefore, studied the lactoferrin content of mature granulocytes in conjunction with the myeloperoxidase and the usual hematological parameters in these two disease classes.

\section{B. Materials and Methods}

In all, 40 normal persons and 54 patients with bacterial infections and 94 with malignant diseases were chosen. Myeloperoxydase was cytochemically determined [2] with o-Tolidin. Lactoferrin was determined by the immunoperoxidase technique according to Stein [3] with Dako reagents purchased from Boehringer Ingelheim Diagnostica. The quantitative evaluation of 


\begin{tabular}{|rc|}
\multicolumn{1}{c}{ date } & MLF - Index \\
\hline 29.9. & 248 \\
30.9. & 239 \\
4.10. & 261 \\
5.10. & 232 \\
6.10. & 242 \\
7.10. & 254 \\
8.10. & 254 \\
9.10. & 260 \\
12.10. & 269 \\
13.10. & 271 \\
& $\bar{x} 253$ \\
& $2 \mathrm{~s} \pm 26$ \\
\hline
\end{tabular}

Fig. 1. Individual biological variation of MLF

variation in myeloperoxidase and lactoferrin content was performed as in the case of ALP, using a score of $0-3$. The $2 s$ range of multiple determinations in one blood sample was found to be \pm 11.4 , and the biological variation among blood specimens taken daily over 14 days, to be \pm 26 (Fig. 1) index points.

Fig. 2. MLF index in $A$ normal persons; $B$ patients with bacterial infections; $C$ patients with malignant diseases and receiving chemotherapy; $D$ patients with kidney transplants and receiving long-term chemotherapy

\section{Results}

The normal persons $(n=40)$ showed a normal distribution with a mean of 231 and a $2 s$ range of \pm 34 . In contrast, the two groups of patients did not show a normal distribution, more than $50 \%$ of their lactoferrin values lying below the $2 \mathrm{~s}$ range of the normal group with mean values of 158 , 189 and 153 for patients with bacterial infections and malignant diseases (Fig. 2), respectively. In patients with bacterial infections, 2-4 days after granulocytosis and left shift a decrease of lactoferrin was noted, which was followed by an increase to the normal values 2 days after normalization of the granulocyte kinetics (Fig. 3). In addition, in patients chronically treated with cytostatic drugs chronically subnormed lactoferrin values were found (Fig. 4).

\section{Discussion}

Our data demonstrate a close correlation between the lactoferrin content of mature granulocytes and the reactive changes with the granulocytic system during bacterial infections. The low lactoferrin values in patients with malignant diseases could be due
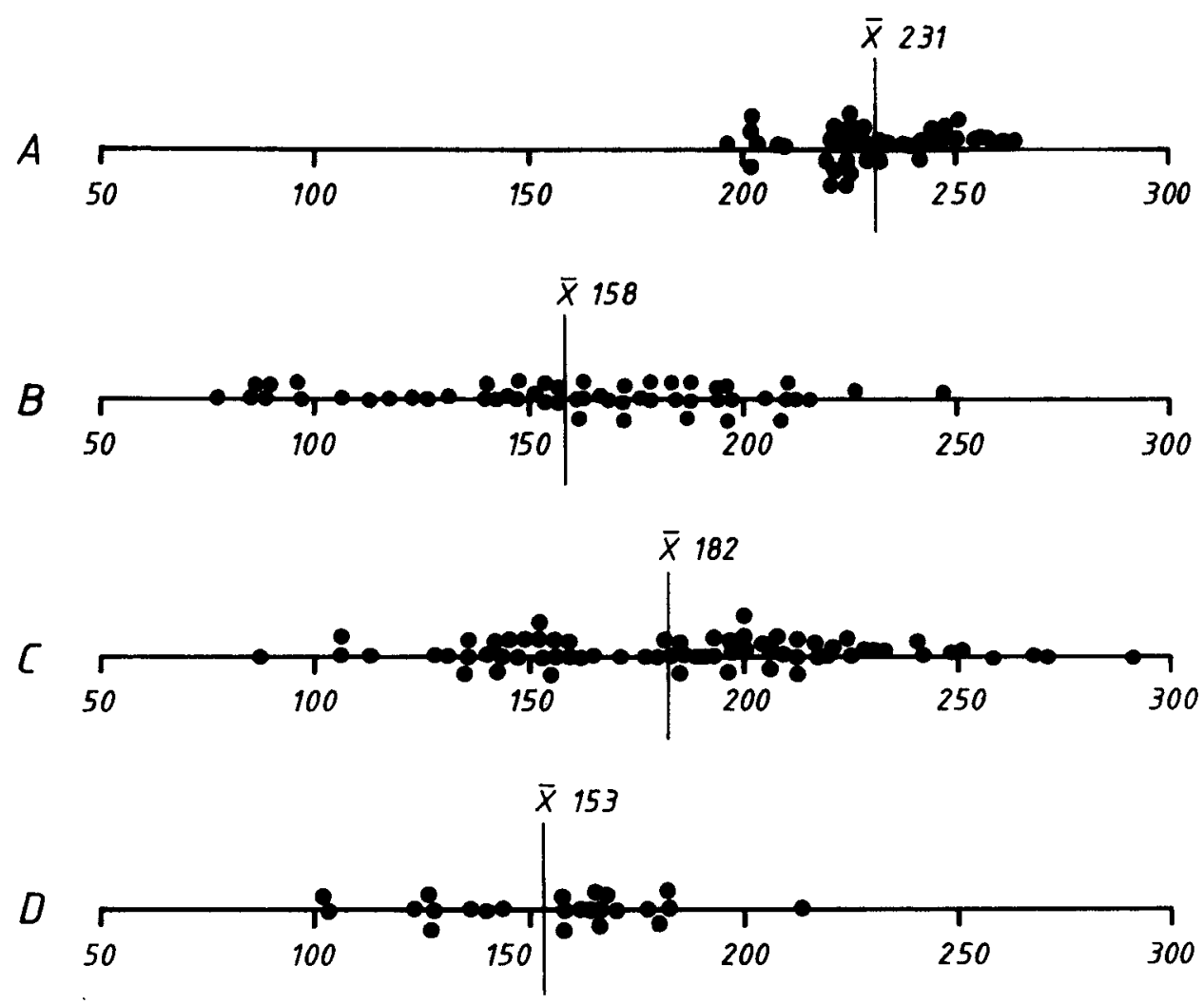


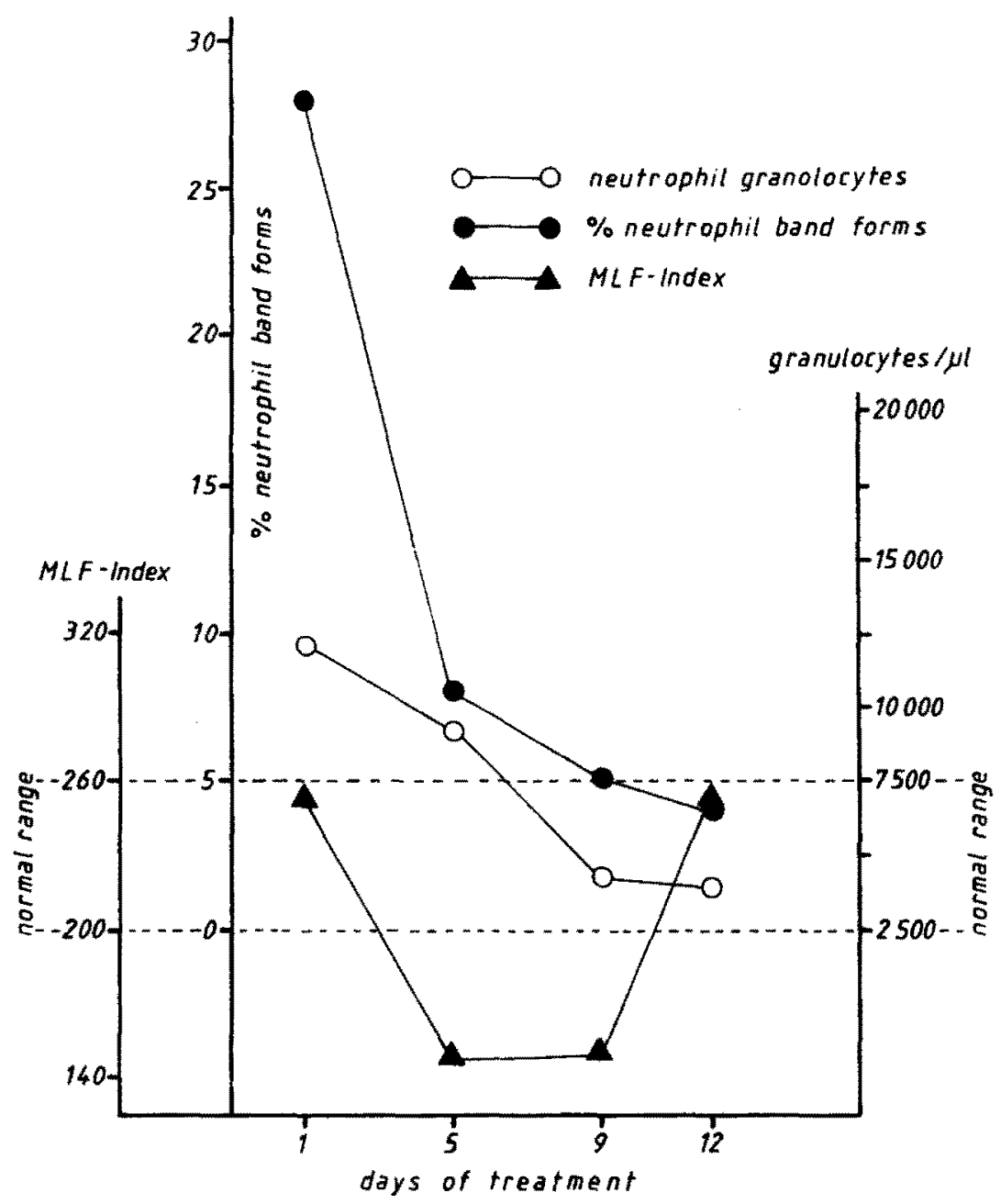

Fig. 3. Follow-up study of a patient with urinary tract infection

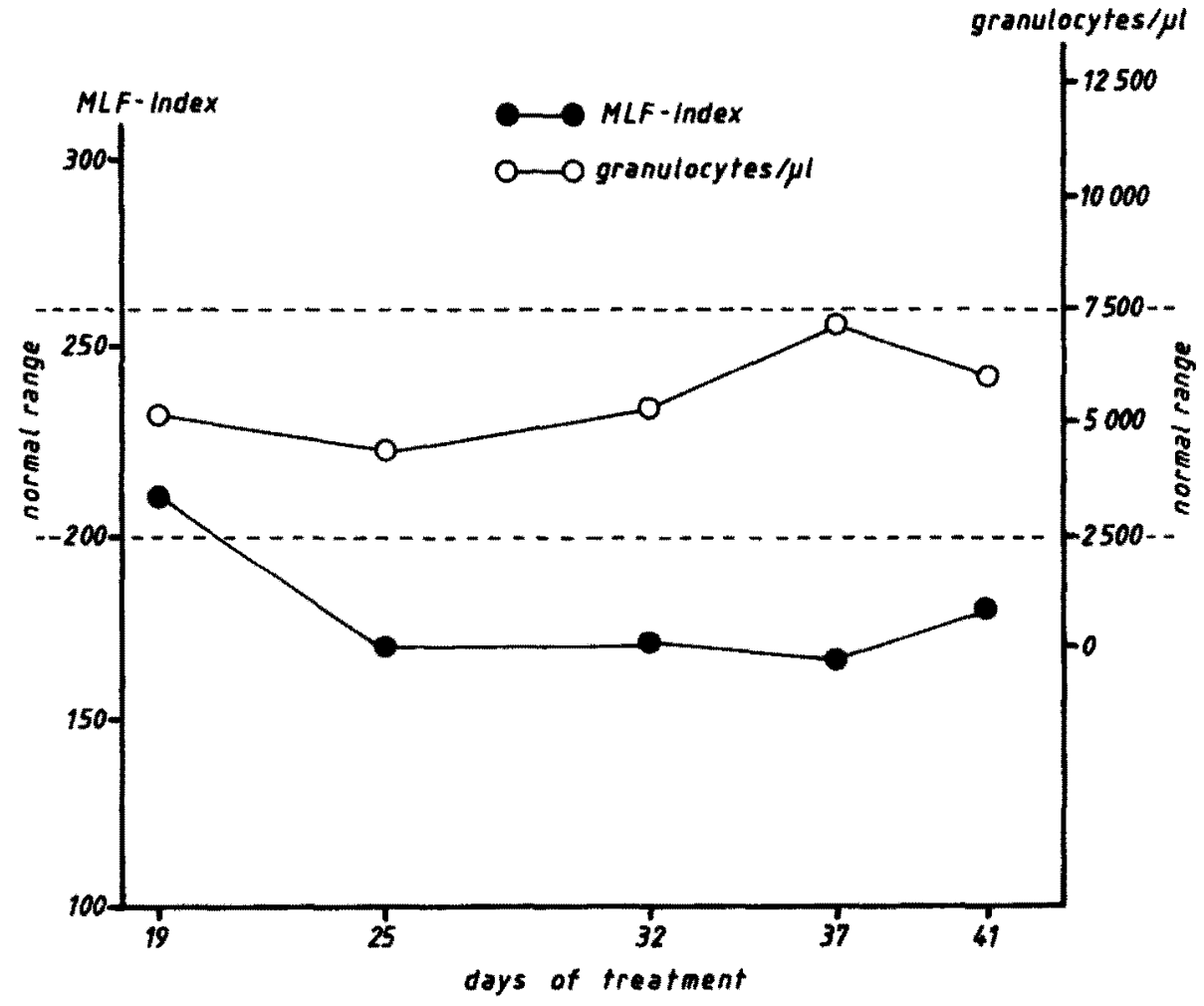

Fig. 4. Follow up study of a patient with a kidney transplant and receiving longterm chemotherapy

to inefficiency of the granulopoietic differentiation. Our data, however, do not support this possibility: Decreased myeloperoxidase levels are known to occur in leukemia and indicate a disturbance of granulopoietic differentiation [4]. In our group of patients with malignant diseases, however, normal myeloperoxidase values were found, and no correlation could be determined between myeloperoxidase and lactoferrin (correlation coefficient below $0.1)$. A disturbance of granulopoietic differ- 


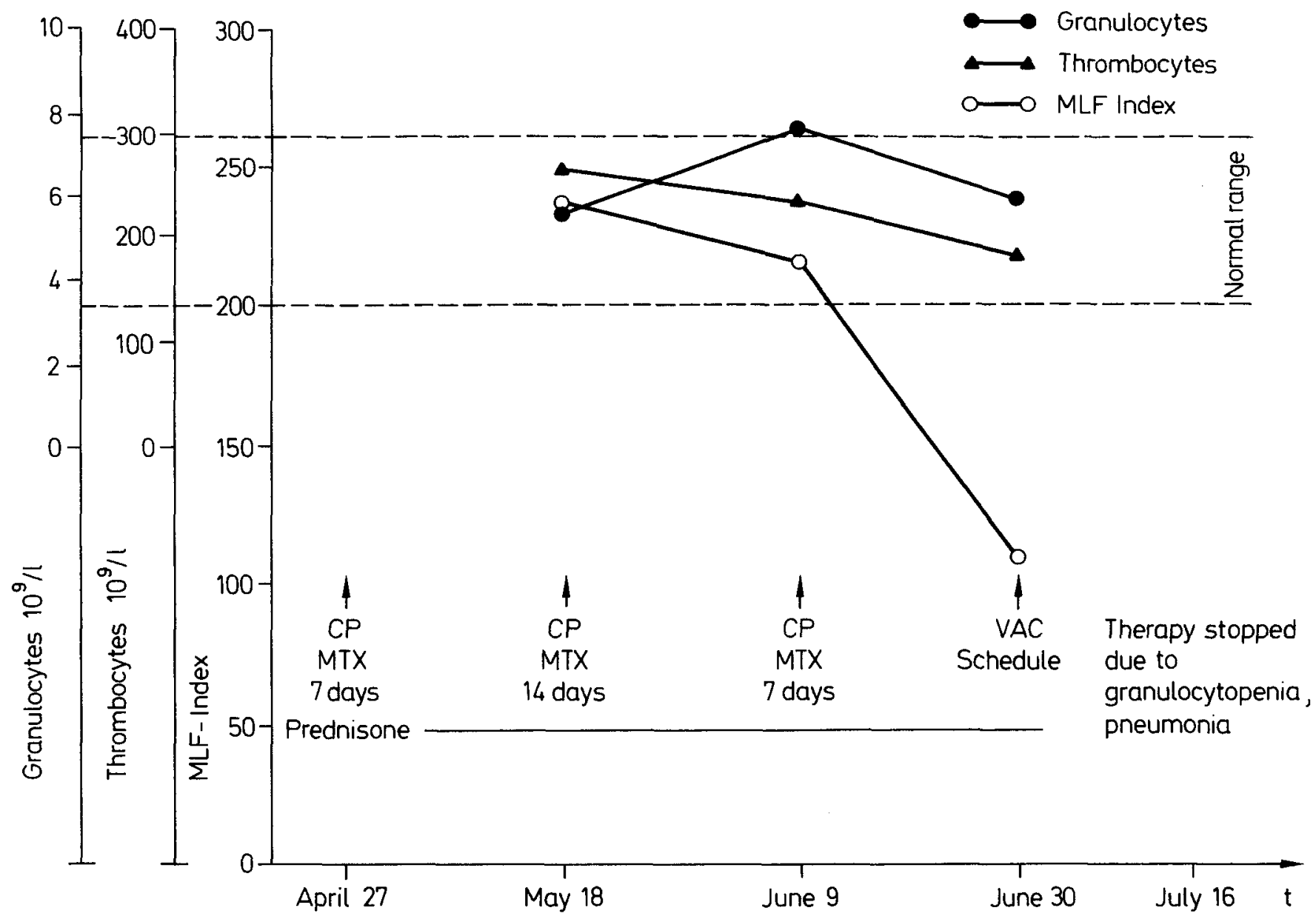

Fig. 5. Follow-up study of a patient with metastatic breast cancer. $C P$ cyclophosphamide; $M T X$ methotrexate; VAC vincristine/adriblastine/ cyclophosphamide

entiation as a cause of the lower lactoferrin values can therefore be excluded in these patients. In both groups of patients it can be assumed that the alteration of the granulopoietic storage pool is followed by a decrease in the lactoferrin content of mature neutrophil granulocytes. Therefore, it seems advisable to study (a) to what extent the immunocytochemical quantitative evaluation of lactoferrin can be used to recognize any toxic damage caused to bone marrow cells by long-term therapies or environmental toxins and (b) whether this would be more sensitive than conventional counting of granulocytes and thrombocytes (Fig. 5).

\section{References}

1. Broxmeyer HE, DeSousa M, Smithyman A, Ralph P, Hamilton J, Kurland JI, Bognacki J (1980) Specifity and modulation of the action of lactoferrin, a negative feedback regulator of myelopoiesis. Blood 55:324

2. Undritz E (1952) Hämatologische Tafeln. Sandoz, Basel, p 28

3. Stein H, Gerdes J, Schwab U, Lemke H, Mason DY, Ziegler A, Schienle W, Diehl V (1982) Identification of Hodgkin and Sternberg-Reed cells as a unique cell type derived from a newly detected small-cell population. Int J Cancer 30:445

4. Rabe K, Rehpenning W, Winkler K, Heinisch B, Krause U, Soltau H, Neth R (1983) Persistent deficiency of myeloperoxidase and lactoferrin in granulopoietic cells of patients with acute leukemia. Springer, Berlin Heidelberg New York Tokio, p 362 (Modern trends in human leukemia, vol 5) 